\title{
The immune response to surgery and infection
}

\author{
ALEKSANDRA M. DĄBROWSKA ${ }^{l}$, ROBERT SŁOTWIŃSKI ${ }^{1,2}$
}

${ }^{1}$ Department of Immunology, Biochemistry and Nutrition, Faculty of Health Sciences, Medical University of Warsaw, Warsaw, Poland ${ }^{2}$ Institute of Experimental and Clinical Medicine of M. Mossakowski, Polish Academy of Science, Warsaw, Poland

\begin{abstract}
Surgical trauma affects both the innate and acquired immunity. The severity of immune disorders is proportional to the extent of surgical trauma and depends on a number of factors, including primarily the basic disease requiring surgical treatment (e.g. cancer), often coexisting infections and impaired nutritional status. Disorder of the immune response following surgical trauma may predispose to septic complications burdened with the highest mortality rate. Extensive surgery in cancer patients is associated with simultaneous activation of pro- and anti-inflammatory processes defined as SIRS (systemic inflammatory immune response) and CARS (compensatory anti-inflammatory immune response). However, it is generally believed that major surgical trauma is accompanied by sustained postoperative immunosuppression, which is particularly important in patients operated on for cancer, since the suppression of the immune system promotes not only septic complications, but also proliferation and tumor metastasis. This paper reviews the main features of immune response to surgical trauma and possibilities of its regulation.
\end{abstract}

Key words: surgical trauma, infection, immune response, SIRS, CARS.

(Centr Eur J Immunol 2014; 39 (4): 532-537)

Several experimental and clinical studies have shown that surgical trauma is associated with disorders of the immune response, in terms of both innate and acquired immunity. The severity of immune disorders is proportional to the extent of surgical trauma and depends on a number of factors, including primarily the basic disease requiring surgical treatment (e.g. cancer), often coexisting infections and impaired nutritional status. It is generally believed that major surgical trauma is accompanied by sustained postoperative immunosuppression, which is particularly important in patients operated on for cancer, since the suppression of the immune system promotes not only septic complications, but also proliferation and tumor metastasis.

\section{Pro- and anti-inflammatory response following surgical trauma}

Local inflammatory response in the surgical wound observed in the early postoperative period is associated mainly with the activation of innate immune mechanisms - the influx of neutrophils and monocytes into the wound and the production of cytokines and chemokines. Local production of proinflammatory mediators increases in the perioperative period, which can potentiate systemic inflammatory response, defined as systemic inflammatory immune response (SIRS) [1]. During initial period after trauma or local infection, inflammatory response is considered as psychological, however, persistent infection of the wound, increasing malnutrition or the re-operation enhance systemic inflammatory response and may lead to multi-organ failure (MOF). The inflammatory response associated with activation of the innate immunity is targeted to elimination of the infectious agent, reducing tissue damage, removal of residues of dead cells and the start of the healing process. Postoperative inflammatory reaction is characterized by massive production of proinflammatory cytokines - interleukin (IL)-1 $\beta$, IL-6 and tumor necrosis factor $\alpha$ (TNF- $\alpha$ ). During pathological conditions (postoperative complications, persistent source of infection), uncompensated excessive and prolonged proinflammatory response in some patients may lead to multiple organ dysfunction syndrome (MODS). However, there are mechanisms maintaining homeostasis of immune response. Research of immunological parameters in patients undergoing surgery show that in the postoperative period increased amounts of anti-inflammatory agents [IL-4, IL-10, sTNFR1, IL-1 receptor antagonist (IL-1Ra), transforming growth factor $\beta$ (TGF- $\beta$ ), cerebrospinal fluid (CSF)] are produced. This phenomenon is considered to be a compensatory response of the immune system, aimed at reducing the severity and duration of the systemic inflammatory response [2]. Compensatory anti-inflammatory immune response (CARS) is a mechanism of adaptation mainly involving acquired (cellular) immunity, including, inter alia, dendritic cells and lymphocytes Treg [3]. The adverse effect of CARS may be prolonged and

Correspondence: Aleksandra Dąbrowska, Medical University of Warsaw, Pawińskiego 3, 02-106 Warszawa, e-mail: aleksandra_dabrowska@op.pl 
increased immunosuppression, predisposing patients to sepsis, secondary infections or late MODS. Initial studies on the subject of systemic inflammatory response to trauma and its compensation based on the belief that these phenomena follow each other - the initial phase of SIRS is followed by CARS. Currently the view of the parallel development of SIRS and CARS response starts to dominate, although these phenomena are still poorly understood, making their clinical interpretation difficult [4]. One example indicating the simultaneous production of anti- and proinflammatory mediators are studies assessing the relationship between the concentration of pro- and anti-inflammatory cytokines and septic complications and operating injuries in surgical cancer patients, published by Mokart et al. [5]. The authors of this paper observed a simultaneous, significant and correlated increase in the concentration of proinflammatory IL- 6 and anti-inflammatory IL-1Ra in the first days after surgery compared to the pre-operative period. In addition, it was reported that IL-6 concentration was significantly higher in the group of patients who developed septic complications, whereas IL-1Ra was significantly higher among patients who developed septic shock. Osuchowski et al. [6] observed a simultaneous production of anti- and proinflammatory cytokines in a murine model of sepsis. In the first five days of sepsis, the growth of IL-6, TNF, IL-1 $\beta, \mathrm{KC}$, macrophage inflammatory protein 2 (MIP-2), monocyte chemoattractant protein-1 (MCP-1) and eotaxin was accompanied by an increasing concentration of soluble TNF receptor (sTNFR), IL-10 and IL-1Ra, which supports the hypothesis of simultaneous pro- and anti-inflammatory reaction during sepsis. Basing on the observations of patients treated in intensive care units (ICU) due to surgical complications, Gentile et al. [7] proposed a new term: persistent inflammation, immunosuppression and catabolism syndrome (PICS) to determine patients who remain in the ICU due to the organ failure, but do not meet the criteria of late MODS. According to the authors, the clinical picture of these patients includes persistent, low intensity inflammation, protein catabolism, loss of lean body mass, impaired wound healing, suppression of the immune system, and recurrent infections. As a result of the prolonged immunosuppression and weight loss, these patients are more prone to nosocomial infections and rarely return to health. Thus, the care of these patients is a major challenge for intensive care units nowadays. Immunological and clinical disorders associated with SIRS, CARS and PICS are presented in Table 1.

\section{Effect of surgical trauma on innate immunity}

The early immune response to the surgical trauma is associated with activation of innate immunity. As the first line of defense, immune cells with phagocytic properties and capability of antigen presentation migrate to the surgical wound. These include neutrophils, macrophages, and dendritic cells. Severe SIRS and infection are accompanied by mobilization of massive amounts of immature neutrophils from the bone marrow into circulation. How-

Table 1. Main clinical and immunological features of SIRS, CARS and PICS. According to [1, 7, 36]

\begin{tabular}{|c|c|c|c|}
\hline & SIRS & CARS & PICS \\
\hline \multirow[t]{6}{*}{$\begin{array}{l}\text { Immunological } \\
\text { disturbances }\end{array}$} & \multirow{6}{*}{$\begin{array}{l}\text { "Cytokine storm" - massive } \\
\text { production of proinflammatory } \\
\text { cytokines: TNF- } \alpha, \text { IL-1, IL- } 6\end{array}$} & $\begin{array}{l}\text { Increased lymphocyte apoptosis, } \\
\text { decreased production of cytokines }\end{array}$ & $\begin{array}{l}\text { Inflammation - changes in } \\
\text { concentrations of IL- } 6 \text {, IL-10, IL-1Ra }\end{array}$ \\
\hline & & $\begin{array}{l}\text { Decreased synthesis of IL- } 1 \beta \text {, IL- } 6 \text {, } \\
\text { IL-8, IL-12, TNF by monocytes in } \\
\text { response to LPS }\end{array}$ & $\begin{array}{l}\text { Monocyte dysfunction - decreased } \\
\text { production of cytokines, decreased } \\
\text { phagocytosis and expression of HLA-DR }\end{array}$ \\
\hline & & $\begin{array}{l}\text { Decreased apoptosis of neutrophils } \\
\text { increased chemotaxis }\end{array}$ & $\begin{array}{l}\text { T-cell anergy - expression of suppressive } \\
\text { molecules, decreased proliferation, shift }\end{array}$ \\
\hline & & Decreased production of IL- $1 \beta$ and & toward $\mathrm{T}_{\mathrm{H}} 2$ response \\
\hline & & IL-1Ra by neutrophils in response to LPS & Increased number of Treg and \\
\hline & & Increased production of IL-10, IL-4 & suppressing activity \\
\hline \multirow{9}{*}{$\begin{array}{l}\text { Clinical } \\
\text { features }\end{array}$} & Body temperature $>38^{\circ} \mathrm{C}$ or $<36^{\circ} \mathrm{C}$ & Skin anergy & ICU stay over 10 days \\
\hline & Pulse $>90$ beats $/ \mathrm{min}$ & Hypothermia & $\mathrm{CRP}>150 \mu \mathrm{g} / \mathrm{dl}$ \\
\hline & $>20$ breaths $/ \mathrm{min}$ & Leukopenia & $\mathrm{TLC}<0,8 \times 10^{9} / 1$ \\
\hline & Hyperventilation $\mathrm{PaCO}_{2}>32$ torr & Susceptibility to infection & Weight loss $>10 \%$ during hospitalization \\
\hline & Leukocyte count $>12,000 / \mathrm{mm}^{3}$ & \multirow{5}{*}{ Difficulties in controlling infection } & or $\mathrm{BMI}<18 \%$ \\
\hline & or $<4,000 / \mathrm{mm}^{3}$ & & Albumins $<3.0 \mathrm{~g} / \mathrm{dl}$ \\
\hline & $>10 \%$ immature neutrophils & & Prealbumins $<10 \mathrm{mg} / \mathrm{dl}$ \\
\hline & & & Retinol binding protein $<10 \mu \mathrm{g} / \mathrm{dl}$ \\
\hline & $\begin{array}{l}\text { *SIRS is diagnosed when at least } \\
\text { two criteria are met }\end{array}$ & & \\
\hline
\end{tabular}

SIRS - systemic inflammatory response syndrome; CARS - compensatory anti-inflammatory response syndrome; PICS - post incarceration syndrome 
ever, Drifte et al. [8], after studying ability of the immature neutrophils to perform an effective immune response (as measured by the ability to survive / perform apoptosis, expression of CD16, Toll-like receptor (TLR) 2, TLR4, CD14, D-2, HLA-DP, and chemotaxis and phagocytosis) in patients with sepsis and SIRS, found that despite its immaturity, these cells are able to fulfill their crucial task from the point of view of innate immunity.

Exposure of immune cells present within infected surgical wounds expressing pattern recognition receptors (PRR) to pathogen-associated molecular patterns (PAMPs) triggers a cascade of reactions, which results in the synthesis of proinflammatory compounds. It was observed that the systemic inflammatory response accompanying operating injuries can also occur without the presence of an infectious agent. This phenomenon can be explained by the discovery of endogenous mediators released during cell destruction - damage-associated molecular patterns or danger-associated molecular patterns (DAMPS) also called alarmins [9]. Alarmins released from damaged tissue are recognized by TLRs expressed by macrophages and dendritic cells. Alarmins can be also recognized by intracellular NOD receptors (nucleotide-binding oligomerization domain-containing protein). Ligation of the alarmin to TLR or NOD receptor activates the signaling pathway leading to the activation of nuclear factor kappaB (NF- $\kappa \mathrm{B})$ and transcription of genes encoding proinflammatory cytokines (IL-1, IL-6, TNF- $\alpha$ ) [10].

\section{Toll-like receptors and surgical infections}

Toll-like receptors are a group of evolutionarily conserved proteins involved in innate immune response. They are present on monocytes, macrophages, neutrophils, dendritic cells and mast cells, however they are also expressed by cells of the adaptive immune response $-\mathrm{T}$ and $\mathrm{B}$ lymphocytes. Changes in the TLRs expression during sepsis and trauma has been shown in the cell culture and animal models [11]. A significant increase in TLR-2 and TLR-4 expression on monocytes obtained from septic patients as compared with healthy controls was observed [12]. However, paradoxically, it was found that an increase in TLRs expression on monocytes during sepsis is accompanied by the decrease in proinflammatory cytokine production [13]. The authors of this study found that mononuclear cells obtained from septic patients with an increased expression of TLRs after ex vivo lipopolysaccharide (LPS) stimulation synthesize significantly less IL- $1 \beta$ than cells derived from patients after surgical trauma and from healthy controls. Interestingly, it was also noted that mononuclear cells obtained from patients after surgical trauma without accompanying sepsis, also show an increased expression of TLR4, but upon stimulation with LPS produce significantly more TNF- $\alpha$ and IL- $1 \beta$ than cells obtained from septic patients and healthy volunteers. It was also shown that LPS stimulation further enhances the expression of TLR receptors on monocytes in surgical patients and in the control group, while in the group of septic patients this phenomenon was not observed. Authors conclude that these observations may indicate reduced ability to respond to the inflammatory secondary signal by peripheral blood mononuclear cells in septic patients. Tsujimoto et al. [11] investigated the relationship between the level of expression of TLR2, TLR4 and CD14 on monocytes and the severity of disease and mortality in sepsis. The authors did not observe any correlation between the expression of these particles and severity of disease pronounced in APACHE II scale (Acute Physiology and Chronic Health Evaluation). It was probably caused by the lack of increase in TLRs expression in patients with a score of APACHE II $>20$ as compared with the patients with less severe sepsis.

\section{Stimulation of Toll-like receptors without the participation of infectious agents - alarmins}

Toll-like receptors may be stimulated without the involvement of infectious agents, by interacting with alarmins. During trauma a number of factors released from the dying and necrotic cells may be a ligand for TLRs. One of the activators of TLR4 is a prosthetic group of hemoglobin - heme. Free heme appears in the extravascular space in large amounts as a result of severe diseases accompanied by hemolysis, hemorrhages or rhabdomyolysis. Figueiredo et al. [14] demonstrated the induction of TNF- $\alpha$ synthesis by macrophages exposed to heme, in a mechanism dependent on myeloid differentiation primary response protein (MyD88), TLR4 and CD14. However, heme did not induce the synthesis of IL-6, which may indicate a different profile of activation of TLR4 than in the case of LPS.

One of the most important alarmins involved in immune response to trauma and infection is non-histone nuclear high mobility group box protein 1 (HMGB1). It can be found in all nucleated mammalian cells where it acts as a transcription factor [15]. High mobility group box protein 1 can get into the extracellular space in the active or passive way. Passive release of HMGB1 into the extracellular space occurs during injury or tissue necrosis and as shown by recent studies, also during apoptosis [16]. Released from damaged cells HMGB1 is involved in the recruitment of macrophages and dendritic cells into the damaged tissue, as well as contributing to the activation of NK cells. Maturation and activation of dendritic cells allows the initiation of a response from T-helper lymphocytes. In the lymph node, mature dendritic cells present antigen complexed with major histocompatibility complex (MHC) molecule to naive T lymphocytes and are the source of HMGB1, further increasing the pool of mature dendritic cells capable of activating naive CD4+, CD8+ and NK cells [17]. Active secretion of HMGB1 by 
immune cells (monocytes, macrophages, dendritic cells) and non-immune cells (endothelial cells, platelets) is a result of exposure to PAMP (such as LPS) and proinflammatory cytokines such as IL-1, TNF [18, 19]. High mobility group box protein 1 is actively secreted as a result of cell exposure to $\mathrm{C} 5 \mathrm{a}$ anaphylatoxin, one of the components of the complement cascade [20]. As stated, produced in the course of experimental sepsis, anaphylatoxin C5a adversely affects survival. C5a induces its proinflammatory actions through interaction with C512 C5aR receptors [21]. Stimulation of C512 receptor with C5a results in an active HMGB1 secretion and further amplifies proinflammatory responses. Blocking $\mathrm{C} 5 \mathrm{a}$ with the specific antibody [22] or Pep inhibitory protein [23] significantly decreases the mortality due to sepsis in an animal model. HMGB1 secretion from activated monocytes/macrophages starts 8-12 hours after stimulation, which is a significantly delayed response in comparison with other proinflammatory factors produced by these cells [24]. As stated, the HMGB1 concentration correlates with the severity of injury, furthermore it has been shown to be significantly higher in patients who developed MODS [25].
When present in the extracellular space, HMGB1 regulates many processes - affects the production of inflammatory cytokines, influences angiogenesis and maturation of dendritic cells, acts as a chemoattractant [26]. The mechanism of its action is associated with stimulation of RAGE (Receptor For Advanced Glycation End Products) and TLRs, including TLR2, TLR4 and TLR9 [27]. The interaction of HMGB1 with RAGE is responsible for its chemotactic properties, whereas interaction with TLR4 induces strong proinflammatory reaction in a mechanism dependent on NF- $\kappa \mathrm{B}$ activation. High mobility group box protein 1 may promote the recruitment of immune cells into the damaged tissue independently of RAGE, by creating heterocomplexes with chemokine CXCL12 and interaction with CXCR4 receptor [28]. Stimulation of TLR4 by HMGB1 initiates a cascade of inflammatory reactions similarly to the LPS stimulation. It requires MyD88 and involves the activation of the transcription factor NF- $\kappa \mathrm{B}$ resulting in the synthesis of proinflammatory cytokines [29]. Toll-like receptor 4 signaling pathway induced by ligation of HMGB1 and/or LPS is presented in Figure 1.

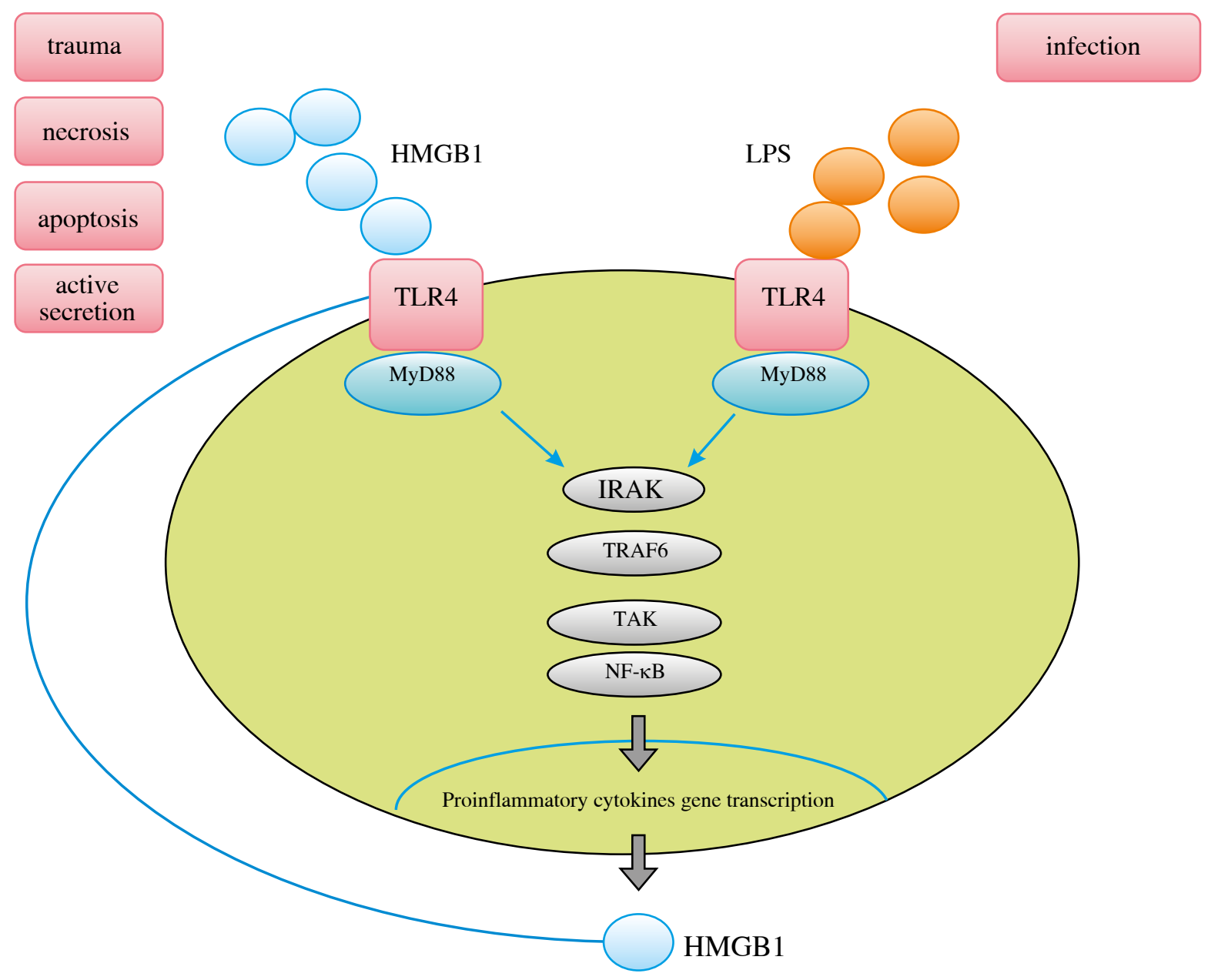

Fig. 1. Toll-like receptor 4 signaling pathway 


\section{Effect of surgical trauma on acquired immunity}

Initiation of specific immune response requires interaction between antigen presenting cells (APC) and $\mathrm{T}$ lymphocytes. In addition to immune cells involved in innate immunity, professional antigen presenting cells such as macrophages and dendritic cells move to injured tissue. Antigens present in injured tissue are taken up by APC and after intracellular processing are presented on their cell surface in a complex with MHC molecules so they can be recognized by $\mathrm{CD} 4+\mathrm{T}$ cells having a specific $\mathrm{T}$-cell receptor (TCR). Depending on the type of antigen and the cytokines interacting with CD4+ cells, they can differentiate towards Th1 or Th2 phenotype. The presence of IL-12 determines the differentiation of Th0 cell toward the Th1 phenotype, while IL-4 promotes differentiation to Th2. Th1 cells by production of interferon $\gamma($ IFN- $\gamma)$ and IL-2 promote primarily a cellular response directed against intracellular pathogens (including viruses) and the elimination of tumor cells. Through the production of IL-4, IL-6 and IL-10, Th2 cells promote humoral immune response directed against extracellular pathogens [30]. As a result of extensive surgical trauma in cancer patients, a shift in Th1/ Th2 balance towards Th2 was observed. Decker et al. [31] showed a decreased IFN- $\gamma$ secretion and increased IL-4 production by peripheral blood polymorphonuclear cells obtained from patients after cholecystectomy, suggesting silencing of cellular responses and enhancing of humoral immune response after a minor surgical procedure. A similar observation was made by Ishikawa et al. [32] however regarding larger surgical trauma (gastrointestinal cancer resection). A significantly reduced $\mathrm{Th} 1 / \mathrm{Th} 2$ ratio observed on the second postoperative day did not return to normal until the fourteenth day after surgery. Additionally, it was observed that in patients who developed postoperative complications, Th1/Th2 ratio remained significantly longer on a lower level as compared to patients without complications, despite the lack of differences on the second day. These reports may suggest that suppression of both Th1 response and intensification of Th2 response may be one of the factors increasing the susceptibility to infections and septic complications. As demonstrated by numerous studies, trauma is associated with an increase in the leukocytes count, but also with a decrease in the number of lymphocytes, both CD4+ and CD8+, which can deepen immunosuppression [33]. One of the mechanisms responsible for the decrease in the number of lymphocytes are disturbances in the mechanisms regulating apoptosis of these cells. It has been shown that prolonged lymphopenia lasting over four days is associated with increased mortality and a higher risk of extended hospitalization [34]. Experimental studies suggest that the prevention of lymphocytes apoptosis by blocking caspase or stimulation of the production of anti-apoptotic Bcl-2 protein might con- tribute to a significant reduction in mortality in patients with severe infections [35].

\section{Conclusions}

Surgical trauma affects both the innate and acquired immunity and disorder of the immune response may predispose to septic complications burdened with the highest mortality rate. Immune disorders associated with extensive surgery in cancer patients are associated with simultaneous activation of pro- and anti-inflammatory processes, and are still a difficult but also an interesting object of study. More detailed knowledge of mechanisms contributing to postoperative immunosuppression and increased susceptibility to septic complications as well as exploring the possibilities of modulating the immune response to trauma is one of the most important goals of experimental and clinical immunology.

The authors declare no conflict of interest. This work was supported by Project No. NN 402306836 founded by Ministry of Science and Higher Education.

\section{References}

1. American College of Chest Physicians/Society of Critical Care Medicine Consensus Conference Committee: American College of Chest Physicians/Society of Critical Care Medicine Consensus Conference. Definitions for sepsis and multiple organ failure and guidelines for the use of innovative therapies in sepsis (1992). Crit Care Med 20: 864-874.

2. Munford RS, Pugin J (2001): Normal response to injury prevent systemic inflammation and can be immunosuppressive. Am J Respir Crit Care Med 163: 316-321.

3. Bone RC, Grodzin CJ, Balk RA (1997): Sepsis: A new hypothesis for pathogenesis of the disease process. Chest 121 : 235-243.

4. Cavaillon JM, Adib-Conquy M, Cloëz-Tayarani I, Fitting C (2001): Immunodepression in sepsis and SIRS assessed by ex vivo cytokine production is not a generalized phenomenon: a review. J Endotoxin Res 7: 85-93.

5. Mokart D, Capo C, Blache JL, et al. (2002): Early postoperative compensatory anti-inflammatory response syndrom is associated with septic complications after major surgical trauma in patients with cancer. Br J Surg 89: 1450-1456.

6. Osuchowski MF, Welch K, Siddiqui J, Remick DG (2006): Circulating cytokine/inhibitor profiles reshape the understanding of the SIRS/CARS continuum in sepsis and predict mortality. J Immunol 177: 1967Y1974.

7. Gentile LF, Cuenca AG, Efron PA, et al. (2012): Persistent inflammation and immunosuppression: A common syndrome and new horizon for surgical intensive care. Trauma Acute Care Surg 72: 1491-1501.

8. Drifte G, Dunn-Siegrist I, Tissičres P, Pugin J (2013): Innate immune functions of immature neutrophils in patients with sepsis and severe systemic inflammatory response syndrome. Crit Care Med 41: 820-832. 
9. Seong SY, Matzinger P (2004): Hydrophobicity: an ancient damage-associated molecular pattern that initiates innate immune responses. Nat Rev Immunol 4: 469-478.

10. Kawai T, Akira S (2007): Signaling to NF-kappaB by Tolllike receptors. Trends Mol Med 13: 460-469.

11. Tsujimoto H, Ono S, Majima T, et al. (2005): Neutrophil elastase, MIP-2, and TLR-4 expression during human and experimental sepsis. Shock 23: 39-44.

12. Armstrong L, Medford AR, Hunter KJ, et al. (2004): Differential expression of Toll-like receptor (TLR)-2 and TLR-4 on monocytes in human sepsis. Clin Exp Immunol 136: 312-319.

13. Tsujimoto H, Ono S, Majima T, et al. (2006): Differential Toll-like receptor expression after ex vivo lipopolysaccharide exposure in patients with sepsis and following surgical stress. Clin Immunol 119: 180-187.

14. Figueiredo RT, Fernandez PL, Mourao-Sa DS, et al. (2007): Characterization of heme as activator of Toll-like receptor 4. J Biol Chem 282: 20221-20229.

15. Andersson U, Erlandsson-Harris H, Yang H, Tracey KJ (2002): HMGB1 as a DNA-binding cytokine. J Leukoc Biol 72: 1084-1091.

16. Scaffidi P, Misteli T, Bianchi ME (2002): Release of chromatin protein HMGB1 by necrotic cells triggers inflammation. Nature 418: 191-195.

17. Lotze MT, Tracey KJ (2005): High-mobility group box 1 protein (HMGB1): nuclear weapon in the immune arsenal. Nat Rev Immunol 5: 331-342.

18. Wang H, Vishnubhakat JM, Bloom O, et al. (1999): Proinflammatory cytokines (tumor necrosis factor and interleukin 1) stimulate release of high mobility group protein-1 by pituicytes. Surgery 126: 389-392.

19. Yu M, Wang H, Ding A, et al. (2006): HMGB1 signals through toll-like receptor (TLR) 4 and TLR2. Shock 26: 174-179.

20. Rittirsch D, Flierl MA, Nadeau BA, et al. (2008): Functional roles for C5a receptors in sepsis. Nat Med 14: 551-557.

21. Cain SA, Monk PN (2002): The orphan receptor C5L2 has high affinity binding sites for complement fragments $\mathrm{C} 5 \mathrm{a}$ and C5a des-Arg74. J Biol Chem 277: 7165-7169.

22. Stevens JH, O'Hanley P, Shapiro JM, et al. (1986): Effects of anti-C5a antibodies on the adult respiratory distress syndrome in septic primates. J Clin Invest 77: 1812-1816.

23. Okada N, Imai M, Okada A, et al. (2012): HMGB1 release by c5a anaphylatoxin is an effective target for sepsis treatment. Clin Exp Pharmacol 2: 2.

24. Schierbeck H, Wähämaa H, Andersson U, Harris HE (2010): Immunomodulatory drugs regulate HMGB1 release from activated human monocytes. Mol Med 16: 343-351.

25. Cohen MJ, Brohi K, Calfee CS, et al. (2009): Early release of high mobility group box nuclear protein 1 after severe trauma in humans: role of injury severity and tissue hypoperfusion. Crit Care 13: R174.

26. Bianchi ME (2009): HMGB1 loves company. J Leukoc Biol 86: 573-576.

27. Park JS, Gamboni-Robertson F, He Q, et al. (2006): High mobility group box 1 protein interacts with multiple Toll-like receptors. Am J Physiol Cell Physiol 290: C917-C924.

28. Schiraldi M, Raucci A, Muńoz LM, et al. (2012): HMGB1 promotes recruitment of inflammatory cells to damaged tissues by forming a complex with CXCL12 and signaling via CXCR4. J Exp Med 209: 551-563.
29. Bell CW, Jiang W, Reich CF 3rd, Pisetsky DS (2006): The extracellular release of HMGB1 during apoptotic cell death. Am J Physiol Cell Physiol 291: C1318-1325.

30. Mosmann TR, Cherwinski H, Bond MW, et al. (1986): Two types of murine helper T cell clone. J Immunol 36: 23482357.

31. Decker D, Schondorf M, Bidlingmaier F, et al. (1996): Surgical stress induces a shift in the type-1/type-2 T-helper cell balance, suggesting down-regulation of cell-mediated immunity commensurate to the trauma. Surgery 119: 316-325.

32. Ishikawa M, Nishioka M, Hanaki N, et al. (2009): Perioperative immune responses in cancer patients undergoing digestive surgeries. World J Surg Oncol $7: 7$.

33. Pagowska-Klimek I, Lewkowicz P, Banasik M, et al. (2007): Isolated head injury in children affects the neutrophil function and lymphocyte count. J Trauma 63: 179-186.

34. Heffernan DS, Monaghan SF, Thakkar RK, et al. (2012): Failure to normalize lymphopenia following trauma is associated with increased mortality, independent of the leukocytosis pattern. Crit Care 16: R12.

35. Hotchkiss RS, Tinsley KW, Swanson PE, et al. (1999): Prevention of lymphocyte cell death in sepsis improves survival in mice. Proc Natl Acad Sci U S A 96: 14541-14546.

36. Ward NS, Casserly B, Ayala A (2008): The Compensatory Anti-inflammatory Response syndrome (CARS) in Critically ill patients. Clin Chest Med 29: 617-625. 